\title{
The effects of three different contrast agents (Gd-BOPTA, Gd-DTPA, and Gd-DOTA) on brachial plexus magnetic resonance imaging
}

\author{
Xiaojing Zhang ${ }^{1,2 \#}$, Wensheng Wang ${ }^{3 \#}$, Tiefang Liu ${ }^{2}$, Yeqing $\mathbf{Q i}^{2}$, Lin $\mathrm{Ma}^{1,2}$ \\ ${ }^{1}$ Medical School of Chinese People's Liberation Army, Beijing, China; ${ }^{2}$ Department of Radiology, The First Medical Centre, Chinese People's \\ Liberation Army General Hospital, Beijing, China; ${ }^{3}$ Department of Radiology, Xiyuan Hospital, China Academy of Chinese Medical Sciences, \\ Beijing, China \\ Contributions: (I) Conception and design: X Zhang, L Ma; (II) Administrative support: W Wang; (III) Provision of study materials or patients: \\ X Zhang, T Liu; (IV) Collection and assembly of data: X Zhang, T Liu, Y Qi; (V) Data analysis and interpretation: X Zhang, L Ma; (VI) Manuscript \\ writing: All authors; (VII) Final approval of manuscript: All authors. \\ \#These authors contributed equally to this study. \\ Correspondence to: Lin Ma. Department of Radiology, The First Medical Centre, Chinese People's Liberation Army General Hospital, Beijing 100853, \\ China. Email: cjr.malin@vip.163.com.
}

Background: MRI is very important for guiding the diagnosis and treatment of brachial plexus diseases. The most used type of MRI brachial plexus imaging is the 3D Short Term Inversion Recovery (STIR) sequence with contrast agent. This study aimed to investigate the effect of three contrast agents; gadobenate dimeglumine (Gd-BOPTA), gadopentetate dimeglumine (Gd-DTPA), and Gadoteric Acid Meglumine (GdDOTA) on brachial plexus magnetic resonance imaging (MRI).

Methods: We recruited 60 patients with suspected brachial plexus injury randomly into three groups. MRI images were obtained from each patient. Prior to scanning, the first group was injected with GD-BOPTA, the second group with Gd-DTPA, and the third with Gd-DOTA. The amount of contrast agent was $0.1 \mathrm{mmol} / \mathrm{kg}$ according to the weight of each patient, the injection rate was $1.5 \mathrm{~mL} / \mathrm{s}$, and $20 \mathrm{~mL}$ saline was injected at the same rate with a high-pressure injector. Immediately after the injection of contrast agent and saline, a 3D Sampling perfection with application optimized contrasts using different flip angle evolutions (SPACE) STIR sequence was used for scanning. The Signal Intensity (SI) and Standard Deviation (SD) of Maximal intensity projection (MIP) images for regions outside the anatomy (ROI background) with area of $17 \mathrm{~mm}^{2}$ on both sides of the C6 peripheral nerves (ROI nerve), and tissue adjacent to the peripheral nerves (ROI tissue) were obtained. Signal to noise ratio (SNR) and contrast to noise ratio (CNR) were then calculated.

Results: The SNR was 40.66 $\pm 25.27,34.65 \pm 14.86$, and 44.63 \pm 30.79 for Gd-BOPTA, Gd-DTPA, and GdDOTA, respectively and the CNR was $20.24 \pm 15.17,16.07 \pm 7.50$, and $20.84 \pm 15.53$ for Gd-BOPTA, GdDTPA, and Gd-DOTA, respectively. In addition, there was no statistical difference in the SNR or CNR of brachial plexus nerves using the three contrast agents to enhance the $3 \mathrm{D}$ SPACE sequence $\chi^{2}=1.877$, $\mathrm{P}=0.391>0.05$ and $\chi^{2}=1.717, \mathrm{P}=0.424$, respectively.

Conclusions: There were no significant differences in the efficacy of three contrast agents in imaging the brachial plexus.

Keywords: Magnetic resonance imaging (MRI); brachial plexus; contrast; signal to noise ratio (SNR); contrast to noise ratio (CNR); Gd-BOPTA; Gd-DTPA; Gd-DOTA; 3D SPACE STIR

Submitted Nov 26, 2020. Accepted for publication Jan 29, 2021.

doi: $10.21037 / \mathrm{atm}-21-348$

View this article at: http://dx.doi.org/10.21037/atm-21-348 


\section{Introduction}

Attaining high-quality images of the brachial plexus has always been problematic for clinicians (1) and several different modalities have been used to address this challenge in daily practice. Although it is safe, ultrasound imaging is greatly affected by the operator, and by bone and lung movement, so the visibility and quality of imaging is poor $(2,3)$. Computed tomography (CT) lacks the contrast resolution required to adequately evaluate soft tissues and the brachial plexus is iso-signal compared to the surrounding muscles and vasculature in images. Sclerotic artifacts from adjacent bones can sometimes interfere with imaging and reduce CT image quality (4) and most importantly, CT uses ionizing radiation. Magnetic resonance imaging (MRI) has many advantages, including it being non-invasive, multi-plane, multi-parameter, its multisequence acquisition and display, and having excellent soft tissue contrast resolution. Therefore, MRI is very important for guiding the diagnosis and treatment of brachial plexus diseases (5-7).

The most used type of MRI brachial plexus imaging is the 3D STIR sequence as after the injection of contrast agent, it is more conducive to the display of brachial plexus nerve fiber bundles $(5,6)$. Combination of MRI regular sequence and 3D STIR sequence could increase the sensitivity of detection of nerve fiber (6). Several magnetic resonance gadolinium-based contrast agents are in regular use and their safety to patients has attracted wide attention. At present, nine kinds of gadolinium-based agents have been approved by the United States Food and Drug Administration (FDA). These can be divided into linear, cyclic, ionic, and non-ionic types according to their chemical structure and charge (8).

Cyclic gadolinium chelates have a lower tendency to free gadolinium and precipitate in the body than linear gadolinium agents and the use of the latter as contrast agents is now banned in Europe. Few study of gadoliniumbased contrast agents focused on brachial plexus imaging. In this study we used the same MRI equipment and inspection sequence to compare three types of gadolinium chelate contrast agents: gadobenate dimeglumine (Gd-BOPTA), gadopentetate dimeglumine (Gd-DPTA) and Gadoteric Acid meglumine (Gd-DOTA).

We present the following article in accordance with the MDAR reporting checklist (available at http://dx.doi. org/10.21037/atm-21-348).

\section{Methods}

\section{Participants}

We enrolled 60 patients with suspected brachial plexus injury who were treated in our hospital from July 2016 to January 2017. The mean age of patients was $47.33 \pm 15.05$ years and there were 32 males and 28 females. Using a random number table, the 60 patients were randomly divided into three groups of 20. The first group was composed of 9 men and 11 women, the second group of 11 men and 9 women and the third group of 12 men and 8 women. The inclusion criteria of patients were as follows: (I) age $\geq 18$ years; (II) patients with a glomerular filtration rate (GFR) $<60 \mathrm{~mL} / \mathrm{min}$; (III) patients who signed informed consent for brachial plexus MRI and contrast agent utility; and (IV) patients who intended to undergo brachial plexus MRI. The exclusion criteria were as follows: (I) age $<18$ years; (II) patients with a GFR $<60 \mathrm{~mL} / \mathrm{min}$; (III) patients with claustrophobia; (IV) pregnancy; (V) patients who had a metal prosthesis which was incompatible with magnetic resonance or could not tolerate magnetic resonance examination for any other reason; (VI) patients who could not tolerate magnetic resonance examination of the brachial plexus. This study was approved by the Ethics Committee of the PLA General Hospital and all patients provided written informed consent. All procedures performed in this study involving human participants were in accordance with the Declaration of Helsinki (as revised in 2013).

\section{MRI scan program and scan parameter settings}

We used a Siemens MR Skyra 3.0T superconducting magnetic resonance scanner (Siemens, Erlangen, Germany; maximum gradient field $45 \mathrm{mT} / \mathrm{m}$, maximum gradient switching rate $200 \mathrm{~T} / \mathrm{m} / \mathrm{s}$ ) which has a 16-channel head coil combined with an 18-channel abdominal coil. Before each examination, patients were checked for contraindications to the examination and informed of its duration. Patients were placed in the in the center of the examination table, in the supine position with the hands placed naturally on both sides of the body and asked to remain still. The positioning line was set at the chin. The scan range covered the brachial plexus and its distal nerve fiber bundles, and the specific scan sequence is shown in Table 1. The sequence scan parameters were as follows: Sag T2: sagittal position, FOV (field of view, FOV) $=26 \mathrm{~cm} \times 26 \mathrm{~cm}$, matrix $=448 \times 312$, 
Table 1 Scanning sequence of the brachial plexus

\begin{tabular}{lll}
\hline $\begin{array}{l}\text { Serial } \\
\text { number }\end{array}$ & Scanning sequence & Position \\
\hline 1 & Sag T2 & Sagittal position T2 \\
2 & Sag T1 & Sagittal position T1 \\
3 & Sag T2 fs dixon & Sagittal position T2 fat pressure \\
4 & Tra T2 msma & Horizontal axis position T2 \\
5 & 3D SPACE STIR & $\begin{array}{l}\text { Coronal position 3D } \\
\text { SPACE STIR }\end{array}$ \\
6 & 3D SPACE STIR + C & $\begin{array}{l}\text { Coronal position 3D SPACE } \\
\text { STIR enhanced }\end{array}$ \\
7 & Sag T1 + C & $\begin{array}{l}\text { Sagittal axis position } \\
\text { T1 enhanced fat pressure }\end{array}$ \\
9 & Cor T1 + C & $\begin{array}{l}\text { Coronal position T1 enhanced } \\
\text { fat pressure }\end{array}$ \\
& & Transverse position \\
10 & Tra T1 fs dixon + C & T1 enhanced fat pressure \\
\hline
\end{tabular}

SPACE, Sampling Perfection with Application-optimized Contrasts by using different flip angle Evolutions; STIR, Short Term Inversion Recovery.

echo time $(\mathrm{TE})=107 \mathrm{~ms}$, repeat time $(\mathrm{TR})=3,500 \mathrm{~ms}$, slice thickness $=3 \mathrm{~mm}$, spacing $=0.5 \mathrm{~mm}$, bandwidth $=260 \mathrm{~Hz} / \mathrm{Px}$, averages $=1$, acquisition time $=1$ minute and 38 seconds.

Sag T1: sagittal position, FOV $=26 \mathrm{~cm} \times 26 \mathrm{~cm}$, matrix $=320 \times 256, \mathrm{TE}=9 \mathrm{~ms}, \mathrm{TR}=600 \mathrm{~ms}$, slice thickness $=3 \mathrm{~mm}$, spacing $=0.5 \mathrm{~mm}$, bandwidth $=260 \mathrm{~Hz} / \mathrm{Px}$, averages $=1$, acquisition time $=1$ minute 41 seconds. Sag T2 fs dixon: sagittal position, FOV $=26 \mathrm{~cm} \times 26 \mathrm{~cm}$, matrix $=320 \times 240$, $\mathrm{TE}=112 \mathrm{~ms}$, $\mathrm{TR}=4,300 \mathrm{~ms}$, slice thickness $=3 \mathrm{~mm}$, spacing $=0.5 \mathrm{~mm}$, bandwidth $=347 \mathrm{~Hz} / \mathrm{Px}$, number of excitations averages $=1$, acquisition time $=1$ minute 39 seconds. Tra T2 msma: transverse position, FOV $=20 \mathrm{~cm} \times 20 \mathrm{~cm}$, matrix $=320 \times 256, \mathrm{TE}=112 \mathrm{~ms}, \mathrm{TR}=6,033 \mathrm{~ms}$, slice thickness $=$ $4 \mathrm{~mm}$, spacing $=0 \mathrm{~mm}$, bandwidth $=284 \mathrm{~Hz} / \mathrm{Px}$, averages $=2$, acquisition time $=2$ minutes and 08 seconds. Sag T1+C: sagittal position, $\mathrm{FOV}=26 \mathrm{~cm} \times 26 \mathrm{~cm}$, matrix $=320 \times 256$, $\mathrm{TE}=10 \mathrm{~ms}$, TR $=650 \mathrm{~ms}$, slice thickness $=3 \mathrm{~mm}$, spacing $=$ $0.5 \mathrm{~mm}$, bandwidth $=347 \mathrm{~Hz} / \mathrm{Px}$, number of excitations averages $=1$, acquisition time $=1$ minute and 50 seconds. Cor T1+C: coronal position, FOV $=26 \mathrm{~cm} \times 26 \mathrm{~cm}$, matrix $=320 \times 256, \mathrm{TE}=10 \mathrm{~ms}, \mathrm{TR}=650 \mathrm{~ms}$, slice thickness $=3 \mathrm{~mm}$, spacing $=0.5 \mathrm{~mm}$, bandwidth $=347 \mathrm{~Hz} / \mathrm{Px}$, averages $=1$, acquisition time $=1$ minute 50 seconds. Tra $\mathrm{T} 1 \mathrm{fs}$ dixon $+\mathrm{C}$ : transverse position, $\mathrm{FOV}=20 \mathrm{~cm} \times 20 \mathrm{~cm}$, matrix $=320 \times 256$,
$\mathrm{TE}=11 \mathrm{~ms}$, $\mathrm{TR}=672 \mathrm{~ms}$, slice thickness $=4 \mathrm{~mm}$, spacing $=0 \mathrm{~mm}$, bandwidth $=347 \mathrm{~Hz} / \mathrm{Px}$, number of excitations $=1$, acquisition time $=3$ minutes and 58 seconds. The sequence used was 3D SPACE STIR and the scanning parameters were as follows: coronal position, FOV $=32 \mathrm{~cm} \times 32 \mathrm{~cm} \times$ $6 \mathrm{~cm}$, matrix $=320 \times 320, \mathrm{TE}=131 \mathrm{~ms}, \mathrm{TR}=3,500 \mathrm{~ms}$, flipping response time TI $=220 \mathrm{~ms}$, slice thickness $=1 \mathrm{~mm}$, bandwidth $=625 \mathrm{~Hz} /$ pixel, acquisition time $=8$ minutes 33 seconds. The contrast medium dosage of the three groups was used in accordance with the principle of $0.1 \mathrm{mmol} / \mathrm{kg}$. The contrast medium was injected through the forearm cubital vein through a high-pressure syringe (Ulrich, Germany), and the injection speed was $1.5 \mathrm{~mL} / \mathrm{s}$. An injection of $20 \mathrm{~mL}$ of saline at a rate to flush the pipeline was used, and the 3D SPACE STIR sequence scan was conducted immediately after the injection of contrast agent and saline. The first group of patients received Gd-BOPTA (Bracco, Italy), the second group Gd-DTPA (Bayer, Germany), and the third group GdDOTA (Jiangsu Hengrui Pharmaceutical Co., Ltd., China).

\section{Image processing and analysis}

Images obtained were transmitted to the Siemens workstation (Siemens Numaris/4 Syngo MR B17) and the maximum intensity projection (MIP) was used to reconstruct the three-dimensional image of the brachial plexus. Two senior radiologists measured the average signal value (SI) and mean square deviation (SD) of the left and right C6 nerve and surrounding soft tissues. The region of interest (ROI) size was 21 pixels. To calculate the average value, we tried to avoid bones, air, and other locations during the measurement. After the ROI was placed, the system software automatically displayed the average SI value and the mean $\mathrm{SD}$. The signal to noise ratio (SNR) and contrast to noise ratio (CNR) were calculated according to Eqs. [1] and [2], respectively.

$$
\begin{aligned}
& \mathrm{SNR}=\mathrm{SI} \text { nerve } / \mathrm{SD} \text { background } \\
& \mathrm{CNR}=(\mathrm{SI} \text { nerve }-\mathrm{SD} \text { tissue }) / \mathrm{SD} \text { tissue }
\end{aligned}
$$

\section{Statistical analysis}

SPSS20.0 software (IBM, Chicago, USA) was used for statistical analysis. The Kolmogorov-Smirnov test was used to determine whether data conformed to a normal distribution. If so, one-way Anova was used and if not, the non-parametric Kruskal-Wallis (KW) test was used, and $\mathrm{P}<0.05$ considered as statistically significant. 


\section{Results}

\section{Normal distribution of the SNR and CNR of the brachial} plexus using three different contrast agents

Both the SNR $(\mathrm{P}=0.007<0.05)$ and $\mathrm{CNR}(\mathrm{P}=0.002<0.05)$,

Table 2 Results of Kolmogorov-Smirnov test

\begin{tabular}{lcc}
\hline Statistics & SNR & CNR \\
\hline Mean & 39.980 & 19.311 \\
Standard deviation & 24.685 & 13.804 \\
Kolmogorov-Smirnov Z & 1.689 & 1.854 \\
P value & 0.007 & 0.002 \\
\hline
\end{tabular}

SNR, signal to noise ratio; CNR, contrast to noise ratio.
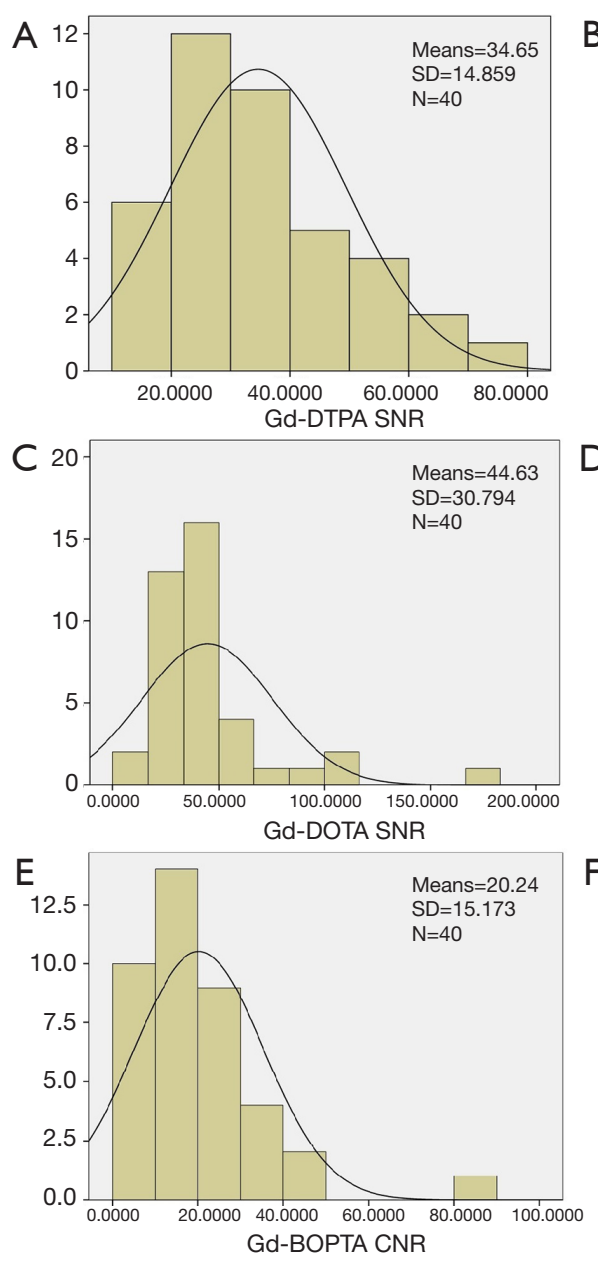

\section{Comparison of the SNR and CNR of the brachial plexus using three different contrast agents}

Comparison of the SNR $\left(\chi^{2}=1.877, \mathrm{P}=0.391\right)$ and $\mathrm{CNR}$ $\left(\chi^{2}=1.717, \mathrm{P}=0.424\right)$ in the three groups indicated that the use of the three contrast agents on the enhanced $3 \mathrm{D}$ SPACE sequence showed no statistical difference (Table 3, Figures 2-4).

\section{Discussion}

Magnetic resonance examination and enhanced magnetic resonance examination are considered safe. In the United
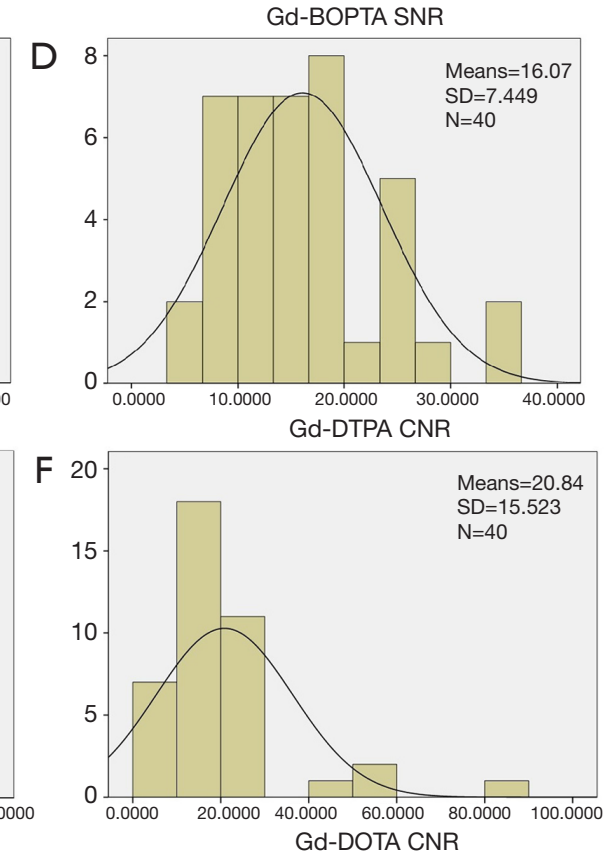

Figure 1 Histogram of SNR and CNR data of the brachial plexus using different contrast agents. (A,D) Using Gd-DTPA; (B,E) using GdBOPTA; (C,F) using Gd-DOTA. These figures show that the above data does not conform to the normal distribution. SNR, signal to noise ratio; CNR, contrast to noise ratio. 
Table 3 SNR and CNR of brachial plexus using different contrast agents

\begin{tabular}{lcc}
\hline Contrast agents & SNR & CNR \\
\hline Gadobeme meglumine & $40.66 \pm 25.27$ & $20.24 \pm 15.17$ \\
Gadopentetate meglumine & $34.65 \pm 14.86$ & $16.07 \pm 7.50$ \\
Gadoterate meglumine & $44.63 \pm 30.79$ & $20.84 \pm 15.53$ \\
$\chi^{2}$ value & 1.877 & 1.717 \\
$P$ value & 0.391 & 0.424 \\
\hline
\end{tabular}

SNR, signal to noise ratio; CNR, contrast to noise ratio.

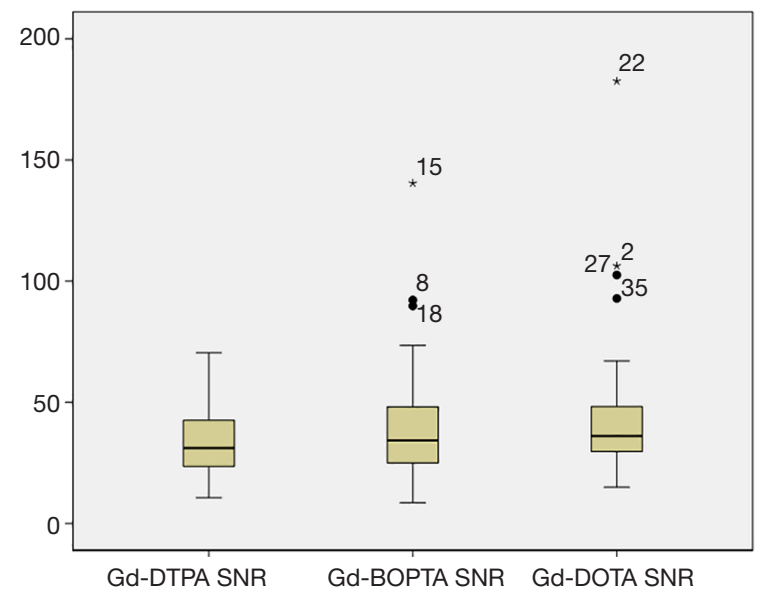

Figure 2 SNR box plot of the brachial plexus after using three contrast agents Gd-DTPA, Gd-BOPTA, and Gd-DOTA. *, Outliers. SNR, signal to noise ratio.

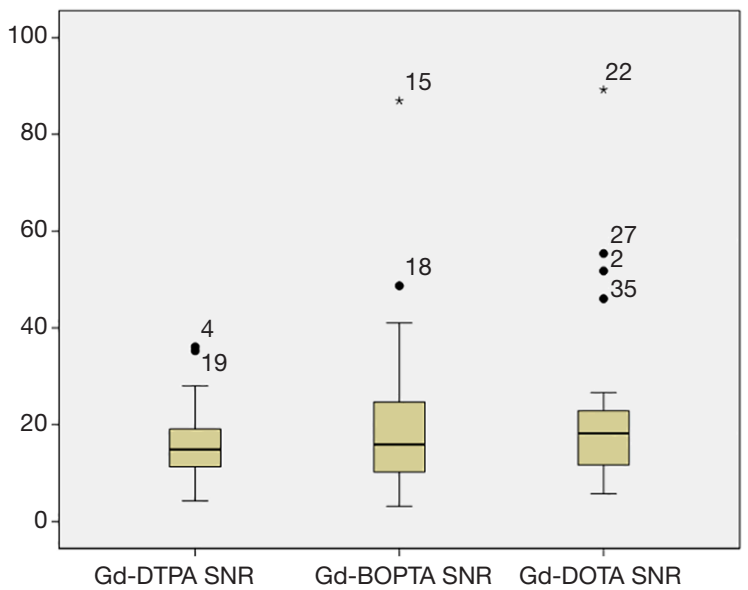

Figure $3 \mathrm{CNR}$ box plot of the brachial plexus after using three contrast agents Gd-DTPA, Gd-BOPTA, and Gd-DOTA. *, Outliers. CNR, contrast to noise ratio.
States, more than 10 million patients undergo enhanced magnetic resonance examination each year, accounting for $40 \%$ to $50 \%$ of all magnetic resonance examinations (9) and both methods are becoming increasingly common in China. Enhanced magnetic resonance examinations are based on the characteristics of the high relaxation rate of gadolinium contrast agents. After the contrast agent is intravenously injected it can not only highlight the shape and boundary of lesions but can also help clinically clarify disease based on the enhanced characteristics of the lesion, greatly benefiting differential diagnosis.

Since MRI contrast agents were first used in 1988, more than 300 million examinations have taken place and currently over 30 million are conducted annually worldwide. On July 21, 2017, the European Medicines Agency (EMA) formally banned the use of some linear gadolinium contrast agents, namely gadopentetate meglumine, gadolinium diamine, and gadolinium fuseamide. It also restricted the use of gadolinium meglumine to liver imaging only. However, one month earlier (May 22, 2017) the U.S. Food and Drug Administration (FDA) issued a statement confirming that the gadolinium deposition in the brain caused by the current MRI gadolinium enhancement was not harmful and did not restrict its use. The FDA statement confirmed it would continue to evaluate the safety of gadolinium contrast agents, and the FDA National Toxicology Research Center was conducting further research.

The heavy metal gadolinium binds to a ligand (chelate). Free $\mathrm{Gd} 3+$ is highly toxic and can replace the $\mathrm{Ca}^{+}$ions on many peptides and biological enzymes in the human body, thereby inhibiting their functions. To reduce its toxicity, researchers have found ways to bind free Gd3+ with various ligands to form stable chelates, which are not easily decomposed, thereby reducing toxicity. Gadolinium contrast agents can be divided into two types, "linear" and "large ring" based on their structure. Linear agents were the first used magnetic resonance contrast agent. Theoretically, the ligand in linear agents is "open ring" and is easy to dissociate. This structure easily separates a certain coordination site in the chelate, and further leads to the sequential separation of other coordination sites, releasing free gadolinium harmful Gd3+. In contrast, in macrocyclic agents, Gd3+ is "fixed" around the ligand making this structure highly stable and not easy to free from the chelating ring structure.

In this study, three representative magnetic resonance contrast agents were selected from several commonly used 

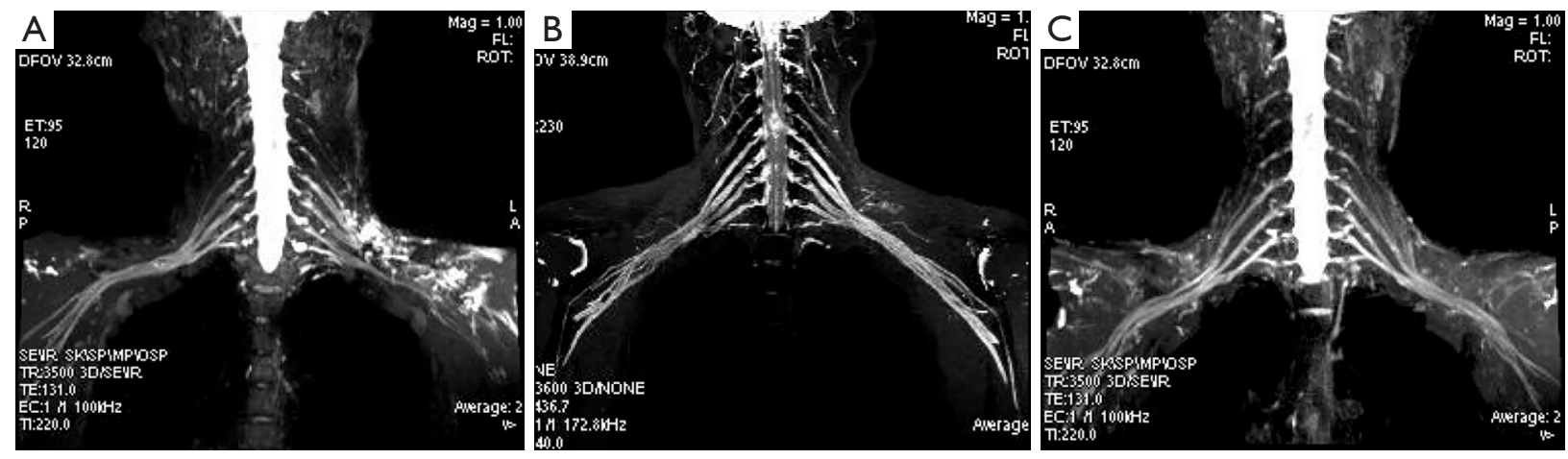

Figure 4 The use of the three contrast agents on the enhanced 3D SPACE sequence. (A) A 39-year-old female patient who presented with a left clavicle fracture and brachial plexus injury. The magnetic resonance contrast agent used during the examination was Gd-BOPTA. (B) A 49-year-old female patient with peripheral neuropathy and the magnetic resonance contrast agent used was gadopentetate meglumine. (C) A 29-year-old male patient presenting with upper limb numbness and the MRI contrast used was Gd-DOTA. It can be seen from the images that the display of the brachial plexus by the three contrast agents is similar.

in our daily work. Gd-BOPTA is a linear contrast agent with high gadolinium concentration and relaxation rate, due to its non-specific distribution of the inter-cells space and the characteristics of specific uptake by hepatocytes. When Gd-BOPTA is injected intravenously, $95 \%$ of the agent is metabolized out of the body by the kidneys and 5\% is excreted through the biliary tract (10), as it holds similar characteristics, Gd-BOPTA can also be used as a specific contrast agent for the liver imaging. Both Gd-BOPTA and Gd-DTPA are ionic linear contrast agents excreted through the kidneys. Gd-DOTA is cyclic contrast agent. There are reports that the stability of gadolinium-based contrast agents can be assigned in the following order: cyclic contrast agent $>$ ionic linear contrast agent $>$ nonionic linear contrast agent (11). While the phenomenon of gadolinium deposition is relatively rare. Studies have shown that their use may result in their deposit in various parts of the human body. As early as 2004, some scholars found that different gadolinium contrast agents had different types of deposition in bones (12) and the brain, especially in the globus pallidus, thalamus, and dentate nucleus (13). Numerous studies have shown that non-ionic linear contrast agents have poor stability and are more likely to cause gadolinium to deposit in the skull (14-18).

The combined use of contrast agents with 3D-T2STIR imaging technology improves the evaluation of arm plexus anatomy and pathology and contributes to the future understanding of brachial plexus neuropathy and the development of surgical planning for surgery (19).

The injection of contrast agent can also improve the ability of 3D SPACE STIR sequencing to display the brachial plexus, by significantly shortening the $\mathrm{T} 1$ relaxation time of tissue and the $\mathrm{T} 2$ relaxation time will be shortened accordingly (20-23). Due to the blood nerve barrier, the contrast agent does not easily enter the nerve sheath, so the normal magnetic resonance signal of the brachial plexus is only slightly affected. Choosing the appropriate T1 imaging parameter will also suppress the lymph nodes, fat, small blood vessels and muscle surrounding the brachial plexus improving its contrast with surrounding tissues.

The SNR and CNR of brachial plexus images after using three contrast agents were compared, and the difference was not statistically significant. Some scholars have reported that when undertaking MR brain examinations using the same dosage condition (24), gadobeme meglumine not only made brain metastases clearer and more obvious, but also increased the detection rate of occult lesions and improved clinical treatment program in comparison to gadopentetate meglumine. However, we found no advantages to applying gadolinium meglumine to examine the brachial plexus, when compared with gadopentetate meglumine and gadoterate meglumine although this may be related to the characteristics of the 3D SPACE STIR sequence.

There are several limitations to this study. Firstly, the sample size was small; secondly, only patients with suspected brachial plexus injury were included; thirdly, the SNR and CNR of only the bilateral $6^{\text {th }}$ cervical nerves were measured; and finally, there is a lack of comparison between machines from different manufacturers and different field strengths. Further studies with larger sample sizes, with 
clearly defined pathologies and evaluating a broader range of brachial plexus nerves using a range of imaging devices and parameters are required to confirm our results.

\section{Conclusions}

There was no significant difference in the efficacy of the three contrast agents, Gd-BOPTA, Gd-DTPA and GdDOTA to evaluate the brachial plexus using enhanced MRI and no adverse reactions were associated with the use of any agent. As the molecular structures of the three contrast agents are different, the stability of the molecular structure of gadolinium meglumine acid is better than that of the other two gadolinium contrast agents. Based on these findings, we recommend the use of gadolinium meglumine acid in enhanced MRI imaging.

\section{Acknowledgments}

Funding: None.

\section{Footnote}

Reporting Checklist: The authors have completed the MDAR reporting checklist. Available at http://dx.doi.org/10.21037/ atm-21-348

Data Sharing Statement: Available at http://dx.doi. org/10.21037/atm-21-348

Conflicts of Interest: All authors have completed the ICMJE uniform disclosure form (available at http://dx.doi. org/10.21037/atm-21-348). The authors have no conflicts of interest to declare.

Ethical Statement: The authors are accountable for all aspects of the work in ensuring that questions related to the accuracy or integrity of any part of the work are appropriately investigated and resolved. This study was approved by the Ethics Committee of the PLA General Hospital and all patients provided written informed consent. All procedures performed in this study involving human participants were in accordance with the Declaration of Helsinki (as revised in 2013).

Open Access Statement: This is an Open Access article distributed in accordance with the Creative Commons Attribution-NonCommercial-NoDerivs 4.0 International
License (CC BY-NC-ND 4.0), which permits the noncommercial replication and distribution of the article with the strict proviso that no changes or edits are made and the original work is properly cited (including links to both the formal publication through the relevant DOI and the license). See: https://creativecommons.org/licenses/by-nc-nd/4.0/.

\section{References}

1. Rehman I, Chokshi FH, Khosa F. MR imaging of the brachial plexus. Clin Neuroradiol 2014;24:207-16.

2. Tagliafico A, Succio G, Serafini G, et al. Diagnostic performance of ultrasound in patients with suspected brachial plexus lesions in adults: a multicenter retrospective study with MRI, surgical findings and clinical follow-up as reference standard. Skeletal Radiol 2013;42:371-6.

3. Martinoli C, Gandolfo N, Perez MM, et al. Brachial plexus and nerves about the shoulder. Semin Musculoskeletal Radiol 2010;15:523-46.

4. Yoshikawa T, Hayashi N, Yamamoto S, et al. Brachial plexus injury: clinical manifestations, conventional imaging findings, and the latest imaging techniques. Radiographics 2006;26:S133-S143.

5. van Es HW, Bollen TL, van Heesewijk HP. MRI of the brachial plexus: a pictorial review. Eur J Radiol 2010;74:391-402.

6. Saifuddin A. Imaging tumours of the brachial plexus. Skeletal Radiol 2003;32:375-87.

7. Amrami KK, Port JD. Imaging the brachial plexus. Hand Clin 2005;21:25-37.

8. Ramalho J, Semelka RC, Ramalho M, et al. Gadoliniumbased contrast agent accumulation and toxicity: an update. Am J Neuroradiol 2016;37:1192-8.

9. Zhou Z, Lu ZR. Gadolinium-based contrast agents for magnetic resonance cancer imaging. Wiley Interdiscip Rev Nanomed Nanobiotechnol 2013;5:1-18.

10. Pasquini L, Napolitano A, Visconti E, et al. GadoliniumBased Contrast Agent-Related Toxicities. CNS Drugs 2018;32:229-240.

11. Bhargava R, Hahn G, Hirsch W, et al. Contrast-enhanced magnetic resonance imaging in pediatric patients: review and recommendations for current practice. Magn Reson Insights 2013;6:95-111.

12. Wiginton CD, Kelly B, Oto A, et al. Gadolinium-based contrast exposure, nephrogenic systemic fibrosis, and gadolinium detection in tissue. AJR Am J Roentgenol 2008;190:1060-8.

13. Zhang Y, Cao Y, Shih GL, et al. Extent of signal 
hyperintensity on unenhanced T1-weighted brain MR images after more than 35 administrations of linear gadolinium-based contrast agents. Radiology 2017;282:516-25.

14. Quattrocchi CC, Mallio CA, Errante Y, et al. Gadodiamide and dentate nucleus $\mathrm{T} 1$ hyperintensity in patients with meningioma evaluated by multiple follow-up contrastenhanced magnetic resonance examinations with no systemic interval therapy. Invest Radiol 2015;50:470-2.

15. Kanda T, Matsuda M, Oba H, et al. High T1 signal intensity in dentate nucleus after multiple injections of linear gadolinium chelates response. Radiology 2015;1:617.

16. Weberling LD, Kieslich PJ, Kickingereder P, et al. Increased signal intensity in the dentate nucleus on unenhanced T1-weighted images after gadobenate dimeglumine administration. Invest Radiol 2015;50:743-8.

17. Kanda T, Osawa M, Oba H, et al. High signal intensity in dentate nucleus on unenhanced T1-weighted MR images: association with linear versus macrocyclic gadolinium chelate administration. Radiology 2015;275:803-9.

18. Ramalho J, Castillo M, AlObaidy M, et al. High signal intensity in globus pallidus and dentate nucleus on unenhanced T1-weighted MR images: evaluation of two linear gadolinium-based contrast agents. Radiology

Cite this article as: Zhang X, Wang W, Liu T, Qi Y, Ma L. The effects of three different contrast agents (Gd-BOPTA, GdDTPA, and Gd-DOTA) on brachial plexus magnetic resonance imaging. Ann Transl Med 2021;9(4):344. doi: 10.21037/atm-21348
2015;276:836-44.

19. Chen WC, Tsai YH, Weng HH, et al. Value of enhancement technique in 3D-T2-STIR images of the brachial plexus. J Comput Assist Tomogr 2014;38:335-9.

20. Veronesi BA, Rodrigues MB, Sambuy MTC, et al. Use of magnetic resonance imaging to diagnose brachial plexus injuries. Acta Ortop Bras 2018;26:131-4.

21. Tagliafico A, Bignotti B, Tagliafico G, et al. Usefulness of IDEAL T2 imaging for homogeneous fat suppression and reducing susceptibility artefacts in brachial plexus MRI at 3.0 T. Radiol Med 2016;121:45-53.

22. Tomura N, Saginoya T, Kokubun M, et al. T2-weighted IDEAL fast spin echo imaging of the brachial plexus: comparison with STIR. Acta Radiol 2015;56:1242-7.

23. Cejas C, Rollán C, Michelin G, et al. High resolution neurography of the brachial plexus by 3 Tesla magnetic resonance imaging. Radiologia 2016;58:88-100.

24. Colosimo C, Demaerel P, Tortori-Donati P, et al. Comparison of gadobenate dimeglumine (Gd-BOPTA) with gadopentetate dimeglumine (Gd-DTPA) for enhanced MR imaging of brain and spine tumours in children. Pediatr Radiol 2005;35:501-10.

(English Language Editor: B. Draper) 